\title{
Nurse-Led Care for Stable Patients with Rheumatoid Arthritis: Quality of Care in Routine Practice Compared to the Traditional Rheumatologist-Led Model
}

\author{
Elena Lopatina (D) - Deborah A. Marshall - Sharon A. Le Clercq • \\ Tom W. Noseworthy · Esther Suter • Carolina De la Rossa Jaimes · \\ Anne Marie Lauf · Dianne P. Mosher · Claire E. H. Barber
}

Received: April 27, 2021 / Accepted: June 16, 2021 / Published online: July 8, 2021

(C) The Author(s) 2021

\section{ABSTRACT}

Introduction: This pragmatic non-inferiority study assessed quality of care within a nurse-led care (NLC) model for stable patients with rheumatoid arthritis (RA) compared to the traditional rheumatologist-led care (RLC) model.

Supplementary Information The online version contains supplementary material available at https:// doi.org/10.1007/s40744-021-00339-3.

E. Lopatina - C. De la Rossa Jaimes

Department of Community Health Sciences, Cumming School of Medicine, University of Calgary, Health Research Innovation Centre (HRIC), 3C60, 3280 Hospital Drive NW, Calgary,

AB T2N 4Z6, Canada

D. A. Marshall ( $\square)$

Department of Community Health Sciences, Cumming School of Medicine, University of Calgary, Health Research Innovation Centre (HRIC), 3C56, 3280 Hospital Drive NW, Calgary,

AB T2N 4Z6, Canada

e-mail: damarsha@ucalgary.ca

\section{S. A. Le Clercq}

Department of Medicine, Cumming School of Medicine, University of Calgary, Health Research Innovation Centre (HRIC), 3C60, 3280 Hospital Drive NW, Calgary, AB T2N 4Z6, Canada

T. W. Noseworthy

Department of Community Health Sciences, Cumming School of Medicine, University of Calgary, Teaching, Research, and Wellness Building (TRW), 3D14-B, 3280 Hospital Drive NW, Calgary, AB T2N 4Z6, Canada
Methods: Data were collected through a chart review. Baseline demographic and clinical characteristics were compared using Chi-square test and $t$ test. The primary outcome measure was the percentage of patients being in remission or low disease activity (R/LDA) with the Disease Activity Score (DAS-28) $\leq 3.2$ at 1-year follow-up. Process measures included the

\section{E. Suter}

Faculty of Social Work, University of Calgary, Health Research Innovation Centre (HRIC), 3C60, 3280 Hospital Drive NW, Calgary, AB T2N 4Z6, Canada

\section{A. M. Lauf}

Cumming School of Medicine, University of Calgary, Health Research Innovation Centre (HRIC), 3C60, 3280 Hospital Drive NW, Calgary, AB T2N 4Z6, Canada

\section{P. Mosher}

Department of Medicine, Cumming School of Medicine, University of Calgary, Health Sciences Centre (HSC), G-802A, 3330 Hospital Dr NW, Calgary, AB T2N 4N1, Canada

\section{E. H. Barber}

Department of Medicine, Cumming School of Medicine, University of Calgary, Health Research Innovation Centre (HRIC), 3AA20, 3330 Hospital Dr NW, Calgary, AB T2N 4N1, Canada

C. E. H. Barber

Department of Community Health Sciences, Cumming School of Medicine, University of Calgary, Health Research Innovation Centre (HRIC), 3AA20, 3330 Hospital Dr NW, Calgary, AB T2N 4N1, Canada 
percentages of patients with chart documentation of (1) comorbidity screening; (2) education on flare management, and (3) vaccinations screening. Outcomes were summarized using descriptive statistics.

Results: Each group included 124 patients. At baseline, demographic and clinical characteristics were comparable between the groups for most variables. Exceptions were the median (Q1, Q3) Health Assessment Questionnaire Disability Index scores $[0(0,0.25)$ in NLC and $0.38(0,0.88)$ in RLC, $p=0.01]$, and treatment patterns with $3 \%$ of NLC and $38 \%$ of RLC patients receiving a biologic agent, $p=0.01$. NLC was non-inferior to RLC with $97 \%$ of NLC and $92 \%$ of RLC patients being in R/LDA at 1-year follow-up. Patients in the NLC group had better documentation across all process measures.

Conclusions: This study provided real-world evidence that the evaluated NLC model providing protocolized follow-up care for stable patients with RA is effective to address patients' needs for ongoing disease monitoring, chronic disease management, education, and support.

Keywords: Delivery of health care; Quality of health care; Health services research; Nurse-led care; Outcome and process assessment; Rheumatoid arthritis

\section{Key Summary Points}

New models of care for stable patients with inflammatory arthritis are proposed as a solution to the challenges of scarce rheumatology healthcare resources.

This study provided real-world evidence that nurse-led care was at least as effective as rheumatologist-led care.

Nurse-led care was also associated with higher documentation of comorbidities screening, education about flares management, and vaccination screening.

Overall, a nurse-led care model where registered nurses provide protocolized follow-up care for stable patients with rheumatoid arthritis represents an effective strategy of addressing the patients' needs for ongoing disease monitoring, chronic disease management, education, and support.

\section{INTRODUCTION}

Rheumatoid arthritis (RA) is a chronic debilitating disease that affects $0.5-1.0 \%$ of the population worldwide $[1,2]$. There are high rates of comorbid conditions in RA including cardiovascular diseases, pulmonary diseases, diabetes, osteoporosis, and depression; all contributing to substantial patient complexity [3, 4]. For optimal outcomes, patients with RA require timely diagnosis and therapy, and life-long specialty care [5-7]. Without continuous rheumatology follow-up, quality of care for patients with RA may decrease, as evidenced by the suboptimal use of conventional synthetic disease-modifying anti-rheumatic drugs (csDMARDs) $[8,9]$. Thus, regular follow-up with a rheumatologist is the current gold standard of care for patients with RA [5-7]. Nonetheless, the capacity of rheumatology care in many regions is insufficient to provide ongoing continuous specialty 


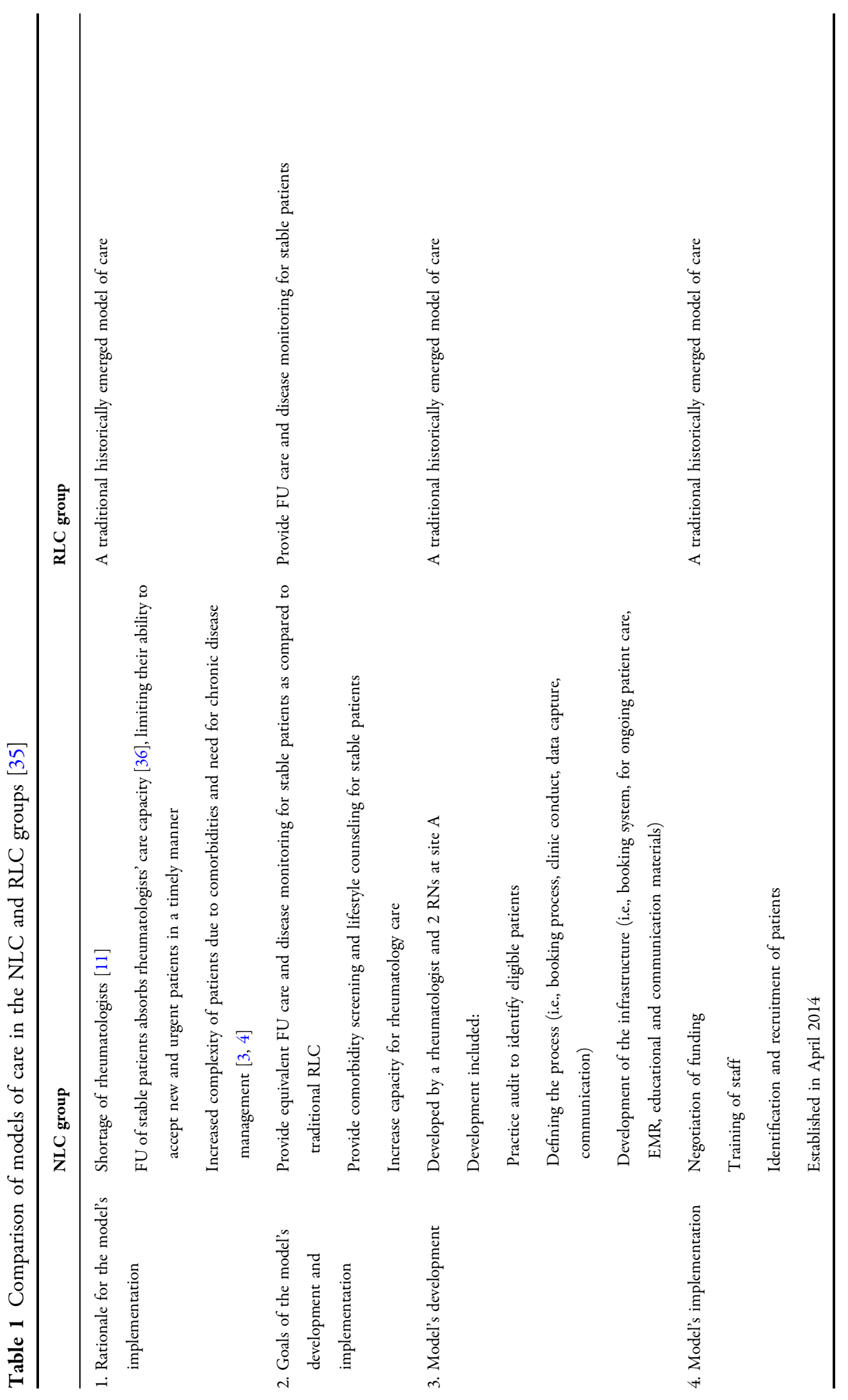




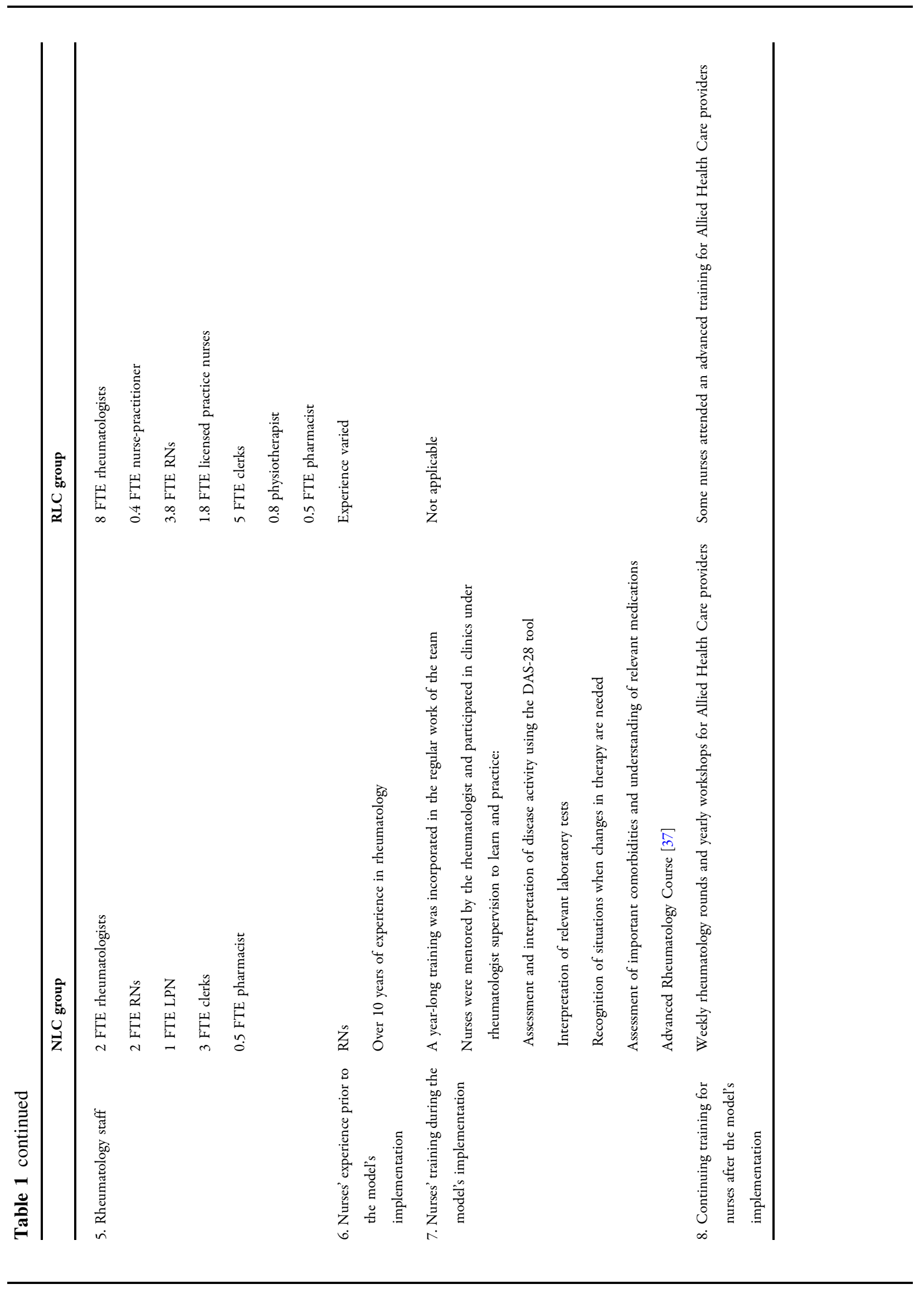




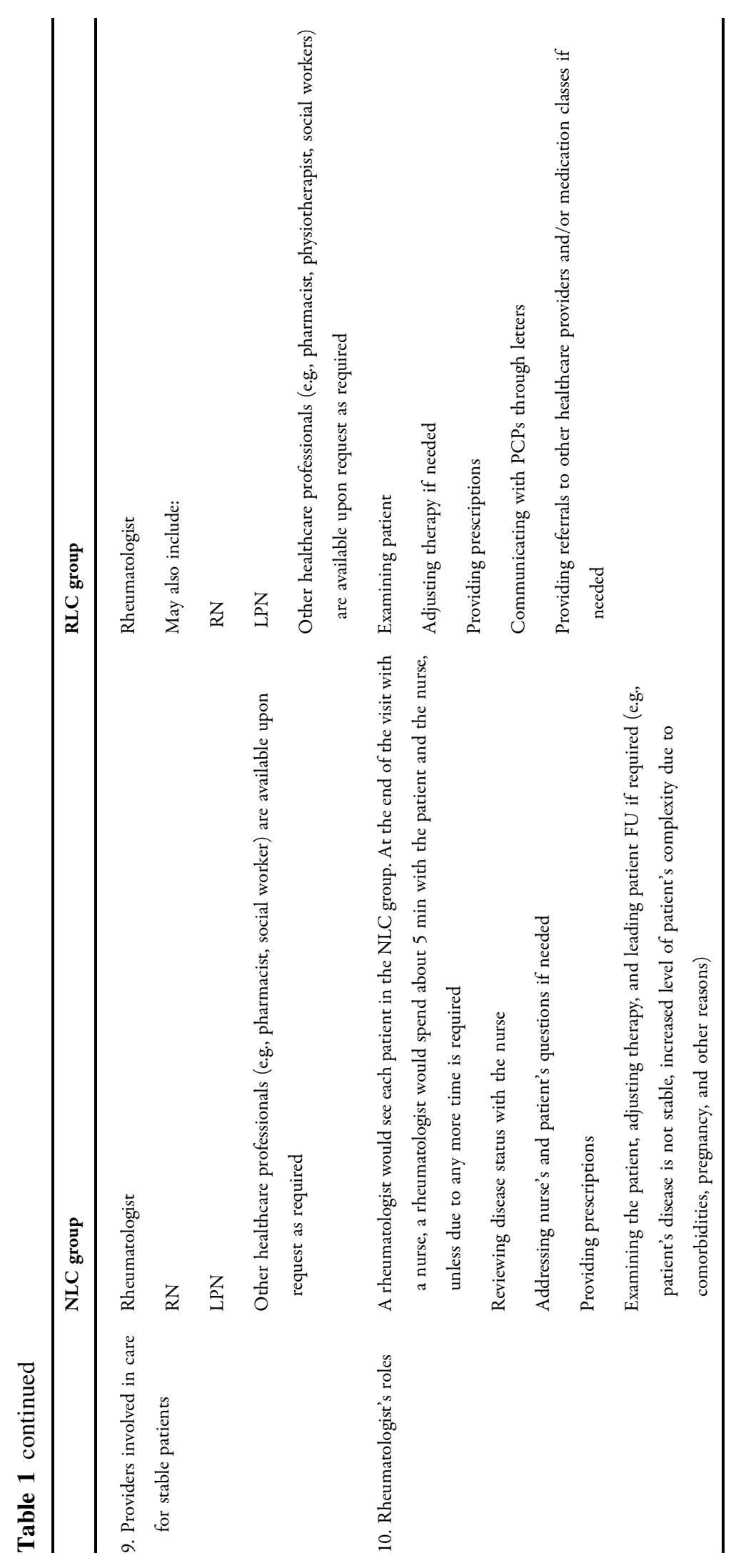




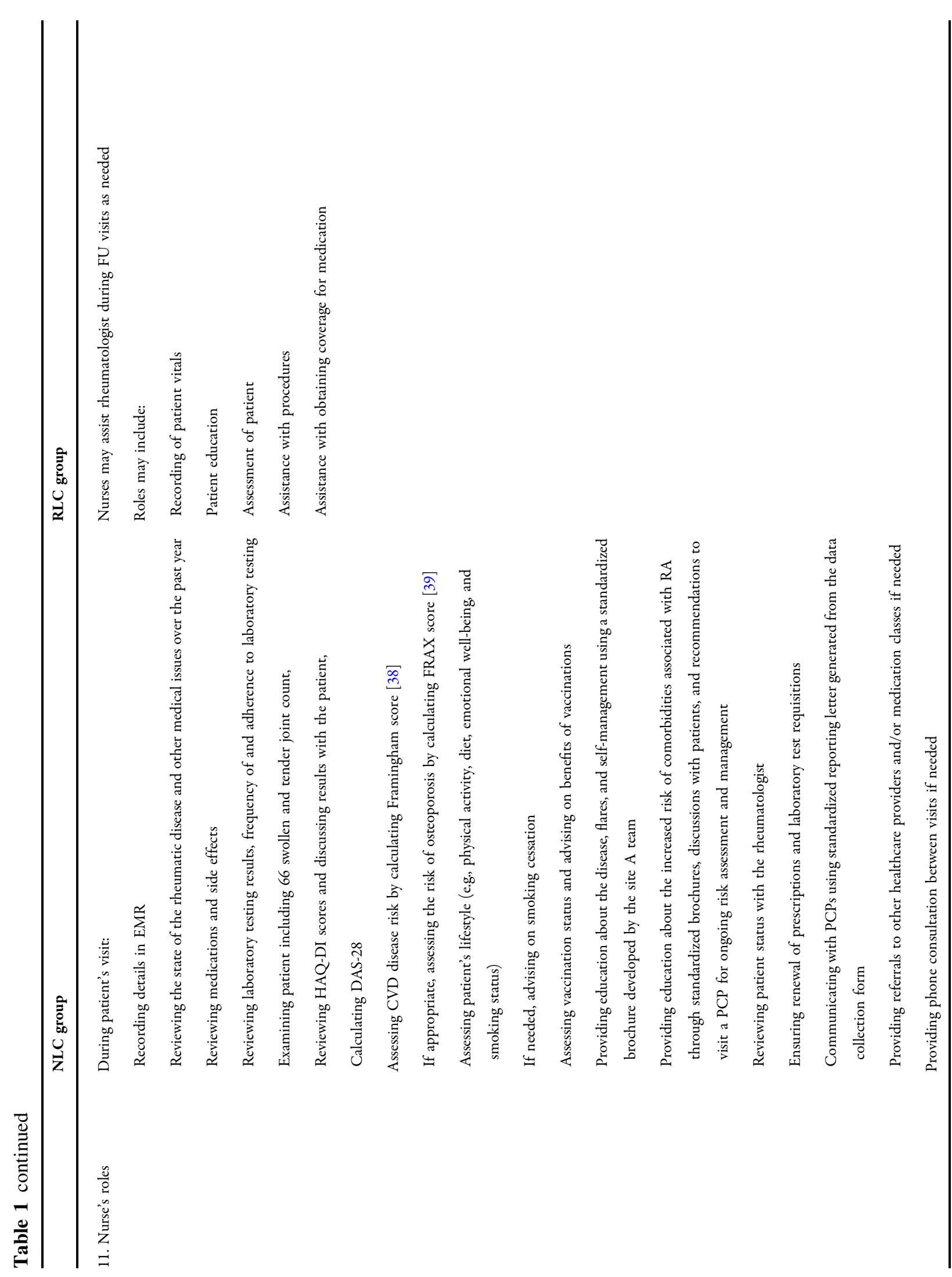




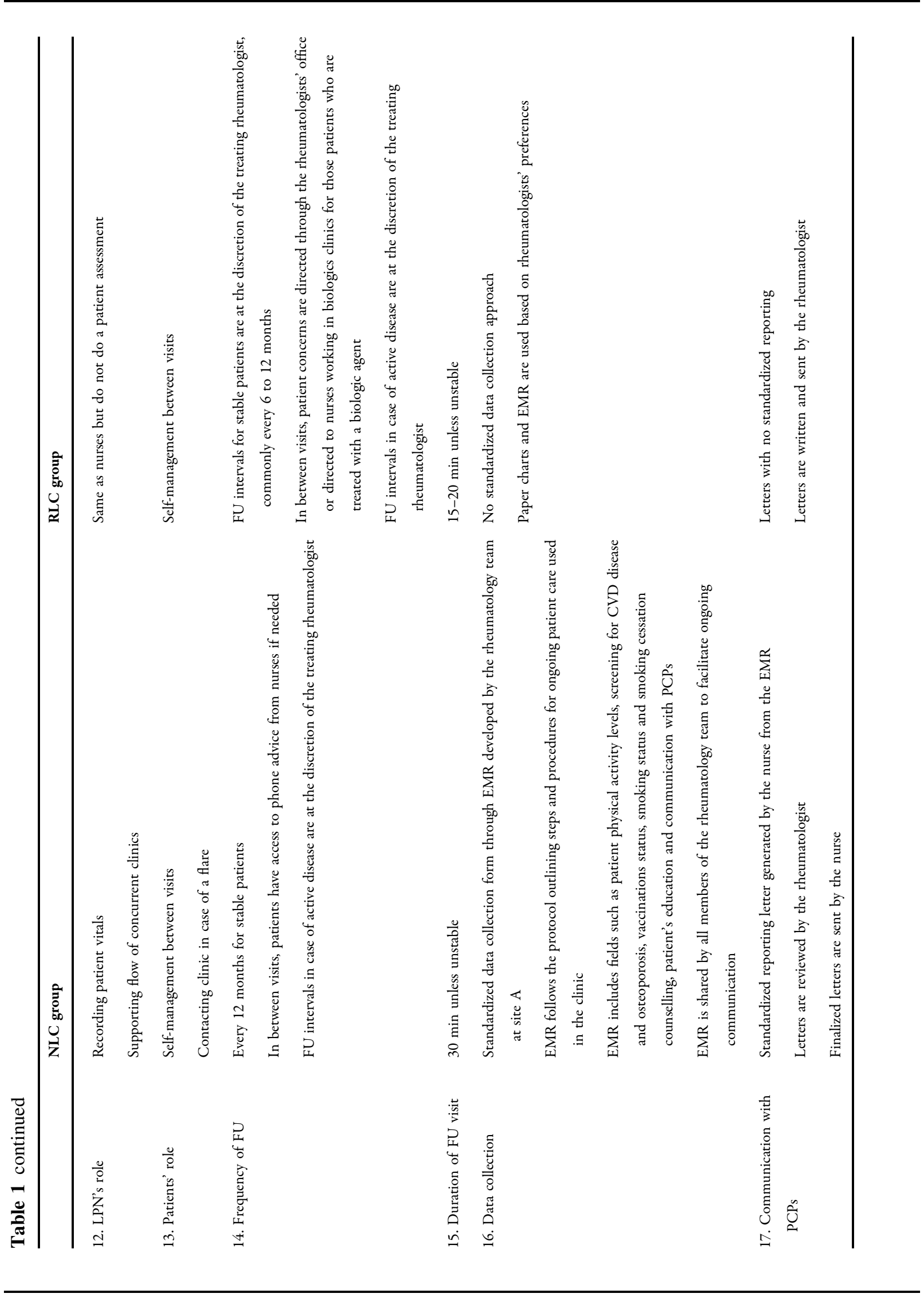


care for established patients and timely access to care for new and urgent patients $[10,11]$.

Innovative models of care delivery have the potential to increase rheumatology care capacity as well as improve the quality of patients' care [12]. Shared care is one example of an innovative model. In shared care, non-physician care providers and/or general medicine physicians with an extended scope of practice work in an alternative role to provide regular patient follow-up in lieu of specialists [13-15]. Besides, they may work in a complementary role to provide additional healthcare services (e.g., screening for comorbidities, psychological support, lifestyle management, and supporting patients in the development of skills and confidence for self-management) [13-15]. For instance, shared care models led by nurses, nurse-led care (NLC), for the follow-up of patients with RA were shown to be at least as effective, safe, and acceptable to patients as compared to rheumatologist-led care (RLC) follow-up [12, 13, 16, 17]. However, the full range of effects of NLC models on quality of care for patients with RA has yet to be explored [13]. Similar to other complex health interventions, NLC affects multiple dimensions of quality of care [13] with some outcomes (e.g., patient knowledge, confidence for self-management, and satisfaction with care) being more difficult to measure $[15,18]$. Research is needed to assess complex effects on the quality of care associated with both alternative and complementary aspects of NLC [15].

This study aimed to assess the quality of care within a collaborative NLC model for stable patients with RA compared to the traditional RLC model using a structured evaluation framework with outcome and process quality measures.

\section{METHODS}

This was a non-inferiority retrospective cohort study at two separate urban rheumatology clinical sites within a single academic center in Alberta, Canada, with a catchment area with over $1,500,000$ people. There are approximately 3,500 and 14,000 patient visits per year at site A and site B, respectively. Both sites offer general 
Table 2 Summary of the assessed clinical characteristics and quality measures

\section{Quality measures}

Full description

Primary outcome measure

Patients in R/LDA at 1-year FU visit

\author{
Patients in R/LDA at 1-year FU visit
}

Secondary outcome measure

Visits where patients were in R/LDA

\section{Clinical characteristics}

HAQ-DI at 1-year FU visit

DAS-28 at 1-year FU visit

Process measures

Visits where patients were not in R/LDA with a change in medications made at that visit

Days to rheumatologist assessment after contacting the rheumatology team about the disease flare

Patients with gaps in care of $>12$ months between 2 consecutive visits

Patients with gaps in care of $>14$ months between 2 consecutive visits

Patients with at least 1 chart documentation on the education about RA flares and when to contact the clinic in case of a flare

Patients with at least 1 chart documentation on the rheumatology team's communication of the associated with RA increased CVD risk to the PCP

Patients with at least 1 chart documentation on their smoking and tobacco use status HAQ-DI at 1-year
FU

DAS-28 at 1-year FU

Not-R/LDA visits with a change in medications

Days to assessment after flare

Gaps in care $>12$ months

Gaps in care $>14$ months

RA flares education

CVD risk communicated to PCP

Smoking and tobacco use status
Visits with R/LDA

Patients in R/LDA at 1-year FU

\author{
Visits with R/LDA
}

\section{Details}

Abbreviated title ${ }^{a}$

Patients who did not have a 1-year FU visit and/or their DAS-28 was not reported or could not be calculated were excluded

Visits where DAS-28 was not reported or could not be calculated were excluded

See details on 'Visits where patients were in R/LDA'

Patients who had an explicit record when they contacted the rheumatology team about the flare were included

Patients who did not have a 1-year FU visit and/or their HAQ-DI score was not reported or could not be calculated were excluded

See details on 'Patients in R/LDA at 1-year FU' 
Table 2 continued

\section{Quality measures}

Full description

Patients who were current smokers or tobacco users with at least 1 chart documentation on the smoking cessation counseling done by the rheumatology team, $\%$

Visits with BP measured and documented in the medical chart, $\%$

Patients whose BP was measured and documented in the medical record at $\geq 80 \%$ of clinic visits, $\%$

Patients $>50$ years with at least 1 chart documentation on the education about the associated with RA increased risk of osteoporosis and/or fractures

Patients with at least 1 chart documentation discussing benefits of vaccinations

Patients with at least 1 chart documentation about being vaccinated with an influenza vaccine

Patients treated with hydroxychloroquine with at least 1 chart documentation on reminders for ophthalmologist yearly FU

\section{Details}

Abbreviated title ${ }^{a}$

Smoking cessation

Patients with explicit records of the smoking cessation counseling, \% counselling (e.g., "Patient was advised about smoking cessation", "Patient is not interested in smoking cessation") were included in the numerator

BP documented, \% Visits of patients with a lower recommended threshold due to diabetes or chronic kidney disease were excluded

BP documented See details on 'BP documented"

at $\geq 80 \%$ of

visits, \%

Education about osteoporosis and/ or fractures

\section{Benefits of} vaccinations discussed

Patients vaccinated with an influenza vaccine

Ophthalmology FU reminders
Patients with explicit recording of patients' education about osteoporosis and/or fractures, their diagnosis and/or treatment (e.g., "risk of osteoporosis and/or fractures was discussed with the patient", "patient was advised to have their bone density scan done", "benefits of calcium and vitamin D were discussed") were included in the numerator

Patients with explicit records of the vaccination status and/or patient's education about vaccinations (e.g., 'patient received annual flu vaccine", "patient refuses to be vaccinated", "benefits of vaccination were discussed", "patient was advised to get a flu shot") were included in the numerator

Patients with explicit records of vaccination status with an influenza vaccine were included in the numerator

Patients with explicit records of ophthalmologist FU and/or the rheumatology team's reminder to book an appointment with an ophthalmologist (e.g., "the patient saw an ophthalmologist a month ago", "the patient was reminded to book a FU appointment with an ophthalmologist”) were included in the numerator

$B P$ blood pressure, $C V D$ cardiovascular disease, $D A S-28$ disease activity score-28, $F U$ follow-up, HAQ-DI Health Assessment Questionnaire Disability Index, \% percentage, $P C P$ primary care provider, $R / L D A$ remission or low disease activity, $R A$ rheumatoid arthritis a Abbreviated titles were used in Table 4 
rheumatology clinics as well as sub-speciality clinics. At site A, stable patients with inflammatory arthritis have a choice to be followed by nurses in NLC clinics, which are offered once or twice a week.

\section{Models of Care Delivery}

The groups of patients included in the study were cared for in two models of care deliveryNLC (site A) and RLC (site B). Characteristics of the models of care are shown in Table 1.

In the NLC model, registered nurses act in an alternative role to monitor patients' disease activity in lieu of a rheumatologist; and in complementary roles to provide patients comorbidity screening and education, as well as to support standardized clinic documentation and communication with primary care providers (PCPs). During patients' visits, nurses follow a protocol outlining steps and procedures for ongoing care. The protocol includes a review of the patient's rheumatic disease and other health conditions over the last year; wellness and lifestyle assessment; rheumatic disease activity assessment; patient education; and, if appropriate, comorbidity screening. Next, a rheumatologist reviews the patient's arthritis status with the nurse, examines the patient if indicated, and renews prescriptions. If the patient's disease is not well controlled, the rheumatologist adjusts treatment as needed and the next appointment would be scheduled in the RLC model. Patients return to the NLC again if they remain stable for at least 6 months. Visit results are recorded in a standardized electronic medical record (EMR) and communicated to the patient's PCP. In between yearly visits, patients have access to phone advice from nurses if needed. In case of a flare, patients are advised to call the clinic and arrange an appointment with a rheumatologist.

Patients in the RLC group were cared for by their rheumatologists at site B. At site B, nursing roles in the follow-up stable patients are not protocolized and vary based on the needs of rheumatologists and availability of staff during clinics. Site B also offers education classes on arthritis, the associated risk of comorbidities, and wellness; however, no standardized protocols for comorbidity screening, patient education, or lifestyle assessment during follow-up visits for stable patients are available. At site B, patient concerns were directed through the rheumatologists' office.

\section{Patient Population}

At site A, patients who would be eligible for the NLC were identified by their treating rheumatologist. Patients were considered to be eligible if they: (1) had no more than 1 visit to the rheumatologist during the previous year; (2) were in remission or low disease activity (R/ LDA) according to Disease Activity Score-28 (DAS-28) for at least 12 months; (3) did not require any changes in medications, and were not treated with oral corticosteroid therapy for RA during the previous visit; (4) did not have multiple unmanaged comorbidities or dementia, and (5) were not pregnant or planning a pregnancy (Supplementary Material). For eligible consenting patients, their next appointments were scheduled in the NLC.

In the present study, the first 124 consecutive patients with RA that (1) were seen in the NLC after April 1, 2014; (2) agreed to continue follow-up in the NLC; (3) were in R/LDA according to DAS-28 at the first visit in the NLC; (4) were not pregnant or planning a pregnancy; and (5) did not move over the follow-up time were included in the analysis.

Patients with RA under RLC at site B were retrospectively identified by applying the criteria used to identify patients eligible for the NLC at site A. First, rosters of patients of two experienced rheumatologists at site $B$ were reviewed. These rheumatologists were selected as they were expected to have a similar mix of patients as the rheumatologist who designed the NLC at site A. Scheduling data were used to identify patients who had no more than one visit to the rheumatologist every 6 months between October 1, 2012 and March 31, 2014 (Supplementary Material). Next, clinical charts of the identified patients were screened to confirm the diagnosis of RA and to ascertain clinical stability at rheumatology visits 1 year before and at the first 
Table 3 Baseline demographic and clinical characteristics for patients in the NLC and RLC care groups and csDMARDs subgroups

\begin{tabular}{|c|c|c|c|c|c|c|}
\hline Characteristics & $\begin{array}{l}\text { NLC, } \\
N=124^{\mathrm{a}}\end{array}$ & $\begin{array}{l}\text { RLC, } \\
N=124^{\mathrm{a}}\end{array}$ & $p$ & $\begin{array}{l}\text { NLC csDMARD, } \\
N=119^{\mathrm{a}}\end{array}$ & $\begin{array}{l}\text { RLC } \\
\text { csDMARD, } \\
N=76^{\mathrm{a}}\end{array}$ & $p$ \\
\hline Age, years, mean (SD) & $60(13)$ & $61(14)$ & 0.51 & $60(13)$ & $62(14)$ & 0.35 \\
\hline Female & $87(70)$ & $83(67)$ & 0.58 & $84(70)$ & $50(65)$ & 0.46 \\
\hline $\begin{array}{l}\text { Disease duration since diagnosis, } \\
\text { years, median }(\mathrm{Q} 1, \mathrm{Q} 3)\end{array}$ & $\begin{array}{l}6(3,10) \\
\quad N=123\end{array}$ & $\begin{array}{l}6(4,15) \\
\quad N=110\end{array}$ & 0.56 & $\begin{array}{c}6(3,10) \\
\quad N=118\end{array}$ & $5(3,8), N=68$ & 0.22 \\
\hline HAQ-DI ${ }^{\mathrm{b}}$, median (Q1, Q3) & $\begin{array}{r}0(0,0.25) \\
\quad N=122\end{array}$ & $\begin{array}{c}0.38(0,0.88) \\
\quad N=100\end{array}$ & 0.01 & $\begin{array}{r}0(0,0.25) \\
N=117\end{array}$ & $\begin{array}{l}0.31(0,0.82) \\
\quad N=56\end{array}$ & 0.05 \\
\hline DAS-28, mean (SD) & $\begin{array}{c}1.77(0.69) \\
N=117\end{array}$ & $\begin{array}{c}1.91(0.66) \\
N=76\end{array}$ & 0.18 & $\begin{array}{c}1.77(0.70) \\
N=114\end{array}$ & $\begin{array}{c}1.84(0.60) \\
N=46\end{array}$ & 0.49 \\
\hline Patients in remission & $\begin{array}{c}103(88) \\
\quad N=117\end{array}$ & $\begin{array}{l}62(82) \\
\quad N=76\end{array}$ & 0.21 & $\begin{array}{l}100(88) \\
\quad N=114\end{array}$ & $39(85), N=46$ & 0.62 \\
\hline Patients in LDA & $\begin{array}{l}14(22) \\
\quad N=117\end{array}$ & $\begin{array}{l}14(28) \\
\quad N=76\end{array}$ & 0.21 & $14(12), N=114$ & $7(15), N=46$ & 0.62 \\
\hline CRP, mean (SD) & $\begin{array}{l}3(5) \\
\quad N=34\end{array}$ & $5(10), N=41$ & 0.51 & $3(4), N=33$ & $3(5), N=28$ & 0.95 \\
\hline ESR, mean $(S D)$ & $\begin{array}{l}12(11) \\
\quad N=85\end{array}$ & $\begin{array}{l}13(15) \\
N=60\end{array}$ & 0.62 & $12(11), N=82$ & $12(16), N=32$ & 0.99 \\
\hline \multicolumn{7}{|l|}{$R A$ treatment } \\
\hline Any csDMARD & $123(99)$ & $103(83)$ & 0.01 & $119(100)$ & $76(100)$ & $\mathrm{n} / \mathrm{a}$ \\
\hline HCQ & $84(68)$ & $66(53)$ & 0.02 & $84(71)$ & $56(74)$ & 0.64 \\
\hline Methotrexate & $112(90)$ & $85(69)$ & 0.01 & $108(91)$ & $63(83)$ & 0.10 \\
\hline Leflunomide & $<6(<5)$ & $<6(<5)$ & 0.10 & $<6(<5)$ & 0 & 0.07 \\
\hline Sulfasalazine & $<6(<5)$ & $18(15)$ & 0.01 & $<6(<5)$ & $13(17)$ & 0.01 \\
\hline $\begin{array}{l}\text { Any combination csDMARD } \\
\text { therapy }\end{array}$ & $80(65)$ & $58(47)$ & 0.18 & $80(67)$ & $49(65)$ & 0.69 \\
\hline Methotrexate + HCQ & $75(61)$ & $51(41)$ & 0.01 & $75(63)$ & $45(59)$ & 0.59 \\
\hline Biologics & $<6(<5)$ & $47(38)$ & 0.01 & $\mathrm{n} / \mathrm{a}$ & $\mathrm{n} / \mathrm{a}$ & $\mathrm{n} / \mathrm{a}$ \\
\hline No csDMARD or biologics & $<6(<5)$ & $<6(<5)$ & $\mathrm{n} / \mathrm{a}$ & $\mathrm{n} / \mathrm{a}$ & $\mathrm{n} / \mathrm{a}$ & $\mathrm{n} / \mathrm{a}$ \\
\hline $\begin{array}{l}\text { Intramuscular or intraarticular } \\
\text { glucocorticoids }\end{array}$ & $<6(<5)$ & $<6(<5)$ & 0.65 & 0 & $<6(<8)$ & 0.21 \\
\hline \multicolumn{7}{|l|}{ Comorbidities and their risk factors ${ }^{b}$} \\
\hline $\mathrm{CVD}^{\mathrm{d}}$ & $9(7)$ & $9(7)$ & $\mathrm{n} / \mathrm{a}$ & $9(8)$ & $8(11)$ & 0.47 \\
\hline Stroke & $<6(<5)$ & $<6(<5)$ & 0.65 & $<6(<5)$ & $<6(<8)$ & 0.33 \\
\hline
\end{tabular}


Table 3 continued

\begin{tabular}{lccclll}
\hline Characteristics & $\begin{array}{l}\text { NLC, } \\
\boldsymbol{N}=\mathbf{1 2 4}^{\mathbf{a}}\end{array}$ & $\begin{array}{l}\text { RLC, } \\
\boldsymbol{N}=\mathbf{1 2 4}^{\mathbf{a}}\end{array}$ & $\boldsymbol{p}$ & $\begin{array}{l}\text { NLC csDMARD, } \\
\boldsymbol{N}=\mathbf{1 1 9}^{\mathbf{a}}\end{array}$ & $\begin{array}{l}\text { RLC } \\
\text { csDMARD, } \\
\boldsymbol{N}=\mathbf{7 6}^{\mathbf{a}}\end{array}$ & $\boldsymbol{p}$ \\
\hline Hypertension & $32(28)$, & $39(33)$, & 0.46 & $31(28), N=110$ & $25(34), N=74$ & 0.42 \\
& $N=114$ & $N=120$ & & & & \\
Diabetes & $9(7)$ & $<6(<5)$ & 0.15 & $7(6)$ & $<6(<8)$ & 0.55 \\
Dyslipidemia & $20(16)$, & $18(15)$, & 0.75 & $18(15), N=118$ & $14(18), N=77$ & 0.56 \\
& $N=123$ & $N=122$ & & & & \\
Obesity (BMI $\left.\geq 30 \mathrm{~kg} / \mathrm{m}^{2}\right)$ & $37(32)$, & $26(27)$, & 0.50 & $35(31), N=112$ & $16(28), N=58$ & 0.62 \\
& $N=117$ & $N=95$ & & & $6(8)$ & 0.01 \\
Osteoporosis & $<6(<5)$ & $12(10)$ & 0.01 & $<6(<5)$ & $<6(<16)$, & 0.41 \\
Current smokers & $19(16)$, & $<6(<11)$, & 0.25 & $19(16)$ & $N=38$ & \\
\hline
\end{tabular}

Values are $n(\%)$ unless otherwise specified

$B M I$ body mass index, CRP C-reactive protein, CVD cardiovascular disease, csDMARD conventional synthetic diseasemodifying anti-rheumatic drug, $D A S$-28 disease activity score-28, ESR erythrocyte sedimentation rate, Q1, Q3 first and third quartiles, $H A Q-D I$ Health Assessment Questionnaire Disability Index, $H C Q$ hydroxychloroquine, $L D A$ low disease activity, $n / a$ not applicable, $N$ number, $N L C$ nurse-led care, $p p$ value, \% percentage, $R A$ rheumatoid arthritis, $R L C$ rheumatologistled care, $S D$ standard deviation

a The numbers of patients included is as described at the top of the table, unless otherwise specified

b Comorbidities and their risk factors at baseline were deemed present at baseline if the condition was documented in the medical record or the patient was treated for the condition and were collected as 'yes', 'no', or 'unknown' if reviewers had major concerns about missing information in the chart

c CVD diseases included myocardial infarction, heart failure, atrial fibrillation, and other arrhythmias

clinical visit after January 1, 2014. If DAS-28 was not reported and could not be calculated, physician notes indicating that the patient was in R/LDA (e.g., "RA stable", "stable disease", "patient is doing well and no changes in medication required") were accepted as an indication of the stable disease. Patients who moved and ceased their follow-up at site B between their first clinical visit after January 1, 2014 and 1-year follow-up visit, as well as patients with multiple unmanaged comorbidities or dementia and pregnant patients, were excluded from the data extraction. The first 124 consecutive patients who met the inclusion criteria were selected for the comparator RLC group.

\section{Duration of Follow-Up}

For the NLC group, the first appointment in the NLC after the clinic's implementation in April, 2014, was used as the baseline visit. For the RLC group, the first clinical visit after January 1, 2014, was used as the baseline visit. In both groups, patients' follow-up continued until their 1-year follow-up visit. The 1-year followup visit was defined as a visit that occurred between 9 and 15 months after the baseline visit. If multiple visits occurred between 9 and 15 months after the baseline, the visit with a longer follow-up time was defined as the 1-year follow-up visit. If no visit occurred between 9 
Table 4 Follow-up characteristics and quality measures assessment for patients in the NLC and RLC groups and csDMARDs subgroups

\begin{tabular}{|c|c|c|c|c|}
\hline & $\begin{array}{l}\text { NLC, } \\
N=124^{\mathrm{a}}\end{array}$ & RLC, $N=124^{a}$ & $\begin{array}{l}\text { NLC csDMARDs, } \\
N=119^{\mathrm{a}}\end{array}$ & $\begin{array}{l}\text { RLC csDMARDs, } \\
N=76^{\mathrm{a}}\end{array}$ \\
\hline FU per patient, days, mean (SD) & $374(25)$ & $397(46)$ & $373(26)$ & $409(44)$ \\
\hline $\begin{array}{l}\text { Visits over } 100 \text { days of FU per } \\
\text { patient, mean (SD) }\end{array}$ & $0.27(0.03)$ & $0.26(0.02)$ & $0.27(0.02)$ & $0.25(0.03)$ \\
\hline \multicolumn{5}{|c|}{ Clinical characteristics and outcome measures } \\
\hline $\begin{array}{l}\text { HAQ-DI at 1-year FU, median (Q1, } \\
\text { Q3) }\end{array}$ & $\begin{array}{r}0(0,0.38) \\
\quad N=117\end{array}$ & $\begin{array}{l}0.44(0,1.13) \\
\quad N=94\end{array}$ & $0.13(0,0.5), N=114$ & $0.5(0,1.13), N=51$ \\
\hline DAS-28 at 1-year FU, mean (SD) & $\begin{array}{l}1.72(0.64) \\
\quad N=113\end{array}$ & $\begin{array}{l}1.93(0.81) \\
\quad N=73\end{array}$ & $1.70(0.63), N=110$ & $1.94(0.86), N=40$ \\
\hline Patients in $\mathrm{R} / \mathrm{LDA}$ at 1 -year FU & $97(109 / 113)$ & $92(67 / 73)$ & $97(107 / 110)$ & $88(35 / 40)$ \\
\hline $\begin{array}{l}\text { Patients who were stable at 1-year FU } \\
\text { b }\end{array}$ & $97(115 / 119)$ & $94(99 / 105)$ & $97(112 / 115)$ & $92(55 / 60)$ \\
\hline Patients in MDA at 1-year FU & $4(4 / 113)$ & $8(6 / 73)$ & $3(3 / 111)$ & $13(5 / 40)$ \\
\hline Visits with R/LDA (7) & $98(234 / 239)$ & $94(186 / 198)$ & $98(228 / 232)$ & $94(106 / 113)$ \\
\hline Visits when patients were stable ${ }^{\mathrm{b}}$ & $94(243 / 258)$ & $91(281 / 310)$ & $95(236 / 248)$ & $92(167 / 182)$ \\
\hline Visits with MDA & $2(5 / 239)$ & $6(11 / 198)$ & $2(4 / 232)$ & $6(7 / 113)$ \\
\hline Visits with HDA & $0(0 / 239)$ & $1(1 / 198)$ & $0(0 / 232)$ & $0(0 / 113)$ \\
\hline \multicolumn{5}{|l|}{ Process measures } \\
\hline $\begin{array}{l}\text { Not-R/LDA visits with a change in } \\
\text { medications }\end{array}$ & $60(3 / 5)$ & $50(6 / 12)$ & $50(2 / 4)$ & $29(2 / 7)$ \\
\hline $\begin{array}{l}\text { Days to assessment after flare, median } \\
\text { (Q1, Q3) }\end{array}$ & $\begin{array}{c}10(5,17) \\
\quad N=7\end{array}$ & $\begin{array}{l}21(\mathrm{n} / \mathrm{a}, \mathrm{n} / \mathrm{a}) \\
\quad N=1\end{array}$ & $12(6,17), N=6$ & $\mathrm{n} / \mathrm{a}$ \\
\hline Gaps in care $>12$ months & $60(74 / 124)$ & $17(21 / 124)$ & $61(72 / 119)$ & $20(15 / 76)$ \\
\hline Gaps in care $>14$ months & $2(3 / 124)$ & $6(7 / 124)$ & $3(3 / 119)$ & $8(6 / 76)$ \\
\hline RA flares education & $98(121 / 124)$ & $2(3 / 124)$ & $98(117 / 119)$ & $0(0 / 76)$ \\
\hline CVD risk communicated to PCP & $90(112 / 124)$ & $2(4 / 124)$ & $91(108 / 119)$ & $4(3 / 76)$ \\
\hline Smoking and tobacco use status & $100(19 / 19)$ & $18(13 / 74)$ & $100(19 / 19)$ & $21(9 / 42)$ \\
\hline Smoking cessation counseling & $84(16 / 19)$ & $80(4 / 5)$ & $84(16 / 19)$ & $75(3 / 4)$ \\
\hline BP documented & $93(239 / 258)$ & $58(182 / 315)$ & $93(229 / 247)$ & $56(105 / 186)$ \\
\hline BP documented at $\geq 80 \%$ of visits & $86(98 / 114)$ & $50(60 / 120)$ & $86(96 / 111)$ & $51(37 / 73)$ \\
\hline $\begin{array}{l}\text { Education about osteoporosis and/or } \\
\text { fractures }\end{array}$ & $92(88 / 96)$ & $19(19 / 101)$ & $85(78 / 92)$ & $0(0 / 60)$ \\
\hline Benefits of vaccinations discussed & $99(123 / 124)$ & $8(10 / 124)$ & $99(118 / 119)$ & $4(3 / 76)$ \\
\hline
\end{tabular}


Table 4 continued

\begin{tabular}{|c|c|c|c|c|}
\hline & $\begin{array}{l}\text { NLC, } \\
N=124^{\mathrm{a}}\end{array}$ & RLC, $N=124^{a}$ & $\begin{array}{l}\text { NLC csDMARDs, } \\
N=119^{\mathrm{a}}\end{array}$ & $\begin{array}{l}\text { RLC csDMARDs, } \\
N=76^{\mathrm{a}}\end{array}$ \\
\hline $\begin{array}{l}\text { Patients vaccinated with an influenza } \\
\text { vaccine }\end{array}$ & $54(67 / 124)$ & $2(3 / 124)$ & $55(65 / 119)$ & $1(1 / 76)$ \\
\hline Ophthalmology FU reminders & $98(83 / 85)$ & $17(12 / 69)$ & $98(83 / 85)$ & $9(11 / 57)$ \\
\hline
\end{tabular}

Values are $\%(\mathrm{~N} / \mathrm{D})$ unless otherwise specified

$B P$ blood pressure, $C V D$ cardiovascular disease, $c$ D DMARD conventional synthetic disease-modifying anti-rheumatic drug, $D A S-28$ disease activity score-28, Q1, Q3 first and third quartiles, $F U$ follow-up, HAQ-DI Health Assessment Questionnaire Disability Index, $H D A$ high disease activity, $L D A$ low disease activity, $M D A$ moderate disease activity, $n / a$ not applicable, $N$ number, $N L C$ nurse-led care, \% percentage (numerator/denominator), $P C P$ primary care provider, $R / L D A$ remission or low disease activity, $R A$ rheumatoid arthritis, $R L C$ rheumatologist-led care, $S D$ standard deviation

a The numbers of patients included is as described at the top of the table, unless otherwise specified

b Based on the analysis where the rheumatology team's opinion about the patient's disease status informed imputations of missing DAS-28 data

and 15 months after the baseline visit, the data collection stopped at 15 months.

\section{Data Sources and Abstraction}

The EMR and paper charts, which included nurses' and rheumatologists' notes, letters to PCPs and other specialists, and laboratory test requests and results, were reviewed. Reviewers with clinical training [international medical graduates (EL, CDRJ) and a nurse (AML)] with previous experience in medical chart reviews abstracted the data. Data were collected using a standardized data abstraction form developed using REDCap electronic data capture tools hosted at the University of Calgary [19, 20]. Each chart was reviewed, and data were abstracted at least by two independent reviewers. Where there were differences in the double data entry, charts were reviewed again and, if needed, discussed with rheumatologists from sites A and B to resolve disagreements.

At baseline, data on disease duration and comorbidities were collected. Comorbidities were deemed present at baseline if the condition was documented in the medical record or the patient was treated for the condition. Data collected at each visit included appointment type; patient's clinical characteristics and laboratory results; medications; documentation about screening for comorbidities and their risk factors; communication with PCPs; and patient education.

\section{Quality Measures Assessment}

Quality measures were selected from national $[21,22]$ and provincial sets of outcomes for the evaluation of models of care for patients with inflammatory arthritis (Table 2).

To capture effects associated with alternative aspects of the evaluated NLC model, the percentage of patients in R/LDA at 1-year follow-up visit [22] and the percentage of clinic visits where patients were in R/LDA over the followup time were selected as the primary and secondary outcome measures, respectively. These outcome measures were identified as key effectiveness outcomes by a provincial arthritis working group tasked with evaluating models of care [23]. Disease activity was extracted from charts or measured with the DAS-28 (erythrocyte sedimentation rate or C-reactive protein (CRP)) depending on data availability. Remission was defined as DAS- $28 \leq 2.6$, low disease activity (LDA) as DAS-28 $>2.6$ and $\leq 3.2$, moderate disease activity (MDA) as DAS- 
Patients in R/LDA at 1-year FU, $\Delta \%(95 \% \mathrm{Cl}):-5 \%(-11 \%, 1 \%)$

Patients in R/LDA at 1-year FU, $\Delta \%(95 \% \mathrm{Cl}):-10 \%(-19 \%,-1 \%)$

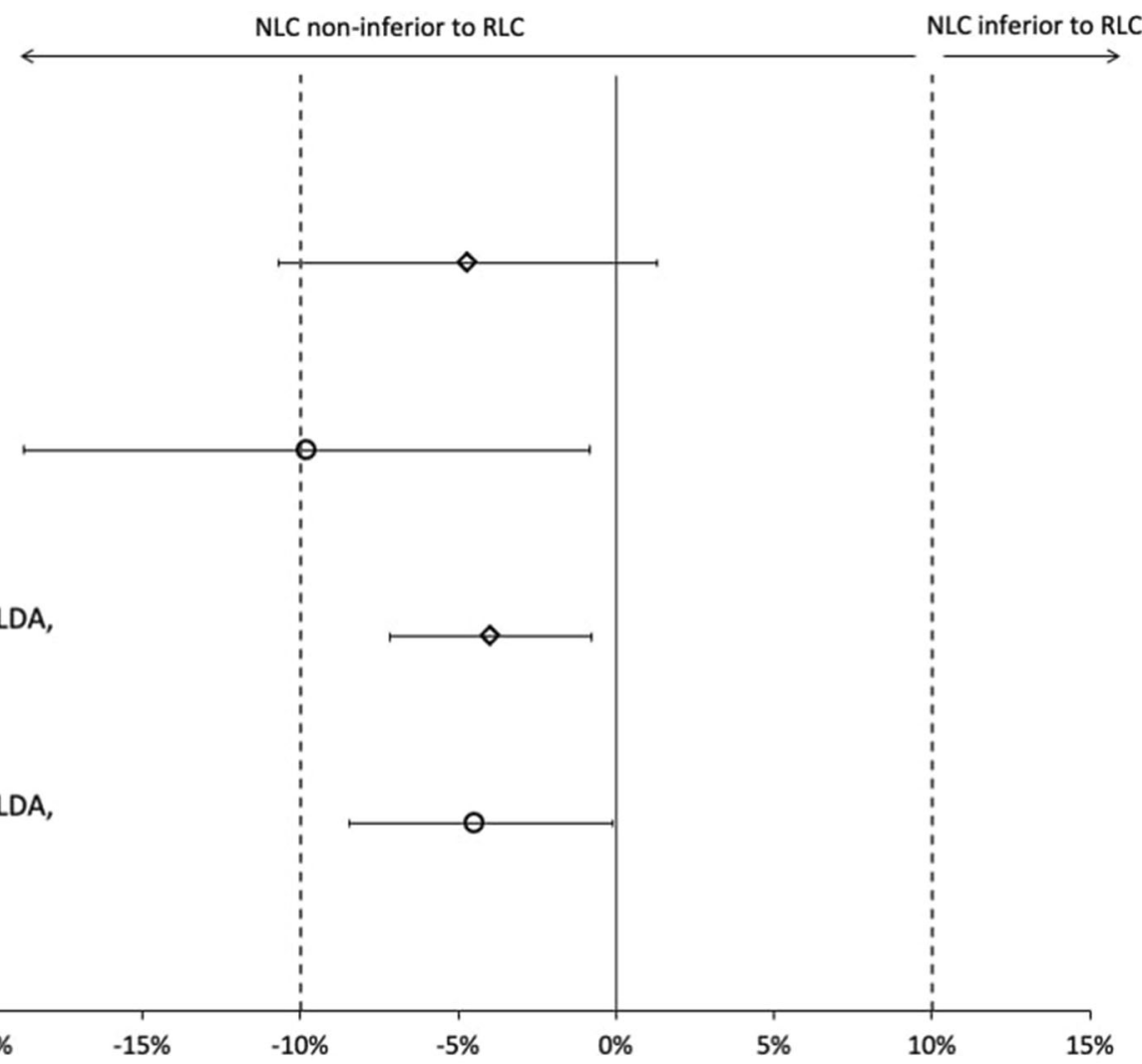

Visits when patients were in R/LDA, $\Delta \%(95 \% \mathrm{Cl}):-4 \%(-7 \%,-1 \%)$

Visits when patients were in R/LDA, $\Delta \%(95 \% \mathrm{Cl}):-4 \%(-8 \%, 0 \%)$

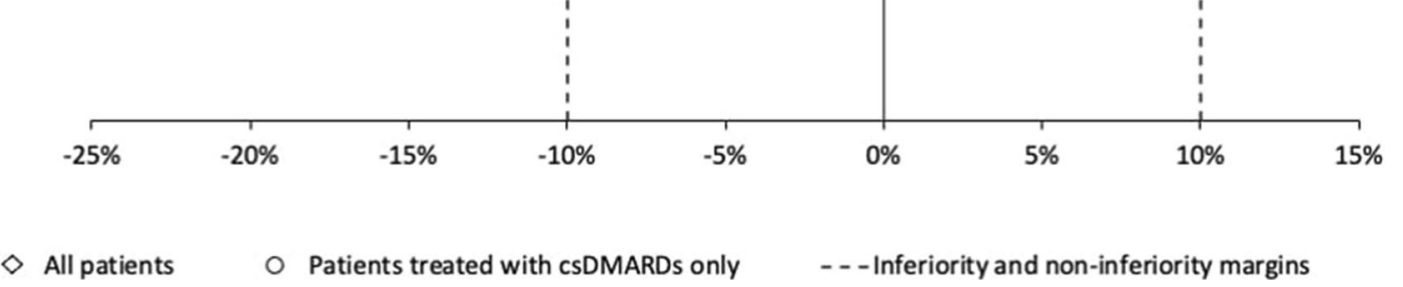

Fig. 1 Forest plot comparing primary and secondary effectiveness outcomes in the NLC and RLC groups and csDMARDs subgroups. $C I$ confidence interval, csDMARD conventional synthetic disease-modifying anti-rheumatic

$28>3.2$ and $\leq 5.1$, and high disease activity (HDA) as DAS-28 > 5.1.

To capture effects associated with complementary aspects of the evaluated NLC model, secondary process measures were used. They included percentages of patients with at least one chart documentation of (1) communication of the associated with RA increased cardiovascular risk to the PCP [21]; (2) smoking status [21]; (3) counseling current smokers or tobacco users to stop smoking [21]; (4) communicating the associated with RA increased risk of osteoporosis and fracture; (5); vaccination status with influenza and pneumococcal conjugate vaccines; (6) discussing benefits of vaccinations with the patient; (7); reminders for ophthalmologist yearly follow-up for patients treated with drug, $F U$ follow-up, $\Delta \%$ mean difference in percentages between RLC and NLC, NLC nurse-led care, \% percentage, $R / L D A$ remission or low disease activity, $R L C$ rheumatologist-led care

hydroxychloroquine; and (8) percentage of patients with blood pressure documented at $\geq 80 \%$ of clinic visits [21].

\section{Sample Size Calculations}

The sample size was calculated using a non-inferiority criterion for the primary outcome of the percentage of patients in R/LDA at 1-year follow-up. For both groups, the percentage 'successes' was assumed to be $90 \%$. Assuming $10 \%$ non-inferiority margin, $80 \%$ power, and one-sided statistical testing with a 5\% significance level, a minimum of 112 patients per group were required. To account for potentially 
missing data, the sample size was increased by $10 \%$ with a total of 248 patients included.

\section{Analysis}

Descriptive statistics [i.e., proportions, means, and their standard deviation (SD) or medians with first and third quartiles (Q1, Q3)], depending on the normality of the data, were used to summarize baseline demographic characteristics, baseline and 1-year follow-up clinical characteristics, and quality measures over the follow-up. Cell sizes with less than 5 patients and/or visits were reported as $<6$. Baseline clinical characteristics for the NLC group patients who were excluded from the analysis due to active disease at the first visit were reported separately (Supplementary Material).

A sensitivity analysis was conducted to account for differences in treatment patterns in the NLC and RLC groups. In this analysis, the subgroups of patients treated with csDMARDs only were included, excluding patients on advanced therapies (i.e., biologic agents) and patients on no medications.

Baseline demographic and clinical characteristics were compared between the NLC and RLC groups and the csDMARDs subgroups using Chi-square test and $t$ test as appropriate for the data. The hypothesis of non-inferiority of NLC compared to RLC in terms of the effectiveness was tested using one-sided 95\% confidence intervals (CIs) of the mean differences in the primary and secondary outcomes between the RLC and NLC groups and between the csDMARDs subgroups [24].

In case of missing data, patients and/or visits were excluded from the analysis (Table 2). For the primary and secondary outcome measures, an additional analysis where the rheumatology team's opinion about the patient's disease status informed imputations of missing disease activity data. In this analysis, patients with missing DAS-28 data were considered to be stable during the visit if there was a note indicating that the patient was stable (i.e., "RA stable", "Disease under control", "The patient is doing well and no changes in medications are required") and were considered to be notstable is the note indicated that (i.e., "RA active", "Flare", "RA is not under control").

\section{Ethics and Consent}

This study was performed in accordance with the Helsinki Declaration of 1964 and its later amendments. The project was approved by the University of Calgary Health Research Ethics Board (REB17-1678). The approval included a waiver of patient consent for this research and publication due to the limited feasibility of obtaining patients' consents for the collection and analysis of retrospective data and the publication of results as well as the minimal risks or discomforts associated with the research.

\section{RESULTS}

\section{Baseline and Follow-Up Characteristics}

A total of 477 charts were screened and 248 patients (124 in the NLC group and 124 in the RLC group) were included in this study (Supplementary Material).

The mean age of patients included was 60.6 years and $68.6 \%$ were female (Table 3 ). At baseline, patients in the NLC and RLC were comparable in terms of their clinical characteristics. The median (Q1, Q3) disease duration since diagnosis was $5.9(2.6,9.8)$ years and 6.1 $(3.8,14.5)$ years and the mean (SD) DAS-28 was $1.76(0.69)$ and $1.89(0.65)$ in the NLC and RLC groups, respectively. The NLC group had a lower median $(\mathrm{Q} 1, \mathrm{Q} 3)$ baseline health assessment questionnaire disability index (HAQ-DI) score $(0(0,0.25)$ than the RLC group $(0.38(0$, $0.88) ; p=0.01)$. The groups also differed in terms of their baseline treatments with $3 \%$ of NLC and $38 \%$ of RLC patients receiving a biologic agent, $p=0.01$. In the sensitivity analysis, the csDMARDs subgroups were comparable in all baseline clinical characteristics. The median (Q1, Q3) baseline HAQ-DI scores were 0 (0, $0.25)$ and $0.31(0,0.82)$ in the NLC and RLC subgroups, respectively; $p=0.05$. 
The observed mean (SD) changes in DAS-28 scores between baseline and 1-year follow-up were $0.12(0.66)$ in the NLC group and 0.21 (0.75) in the RLC group. The mean (SD) changes in HAQ-DI were - $0.17(1.26)$ in the NLC group and 0.05 (0.39) in the RLC group. These changes were below thresholds reported in the literature for minimal clinically important changes of 1.2 for deterioration in DAS-28-CRP and -0.38 for improvement in HAQ-DI $[25,26]$. In the sensitivity analysis, there were no clinically significant differences in the observed changes in DAS-28 and HAQ-DI scores between baseline and the 1-year follow-up in either csDMARD subgroup.

\section{Quality Measures Assessment}

DAS-28 scores were available or could be calculated to assess the primary outcome measure in $92 \%$ of NLC patients and 59\% of RLC patients (Table 4). Overall, five NLC and 12 RLC patients were not in R/LDA during at least one visit over the follow-up time. Out of those, 16 patients were in MDA and one RLC patient was in HDA during one follow-up visit. The mean (SD) number of visits over 100 days of follow-up was $0.27(0.03)$ and $0.26(0.02)$ per patient in the NLC and RLC groups, respectively (Table 4). For patients who were not in remission or low disease activity during at least one visit over the follow-up time, the mean (SD) number of visits over 100 days of follow-up was $0.71(0.29)$ in the NLC group and $0.81(0.22)$ in the RLC group.

Patients were in R/LDA during the majority of visits with $97 \%$ of NLC patients and $92 \%$ of RLC patients being in R/LDA at the 1-year follow-up (Table 4). The mean difference (95\% CI) in DAS-28 between NLC and RLC groups at 1 -year follow-up was $0.21(-0.39,-0.03)$. In the sensitivity analysis, $97 \%$ of NLC patients and $88 \%$ of RLC patients were in R/LDA at the 1-year follow-up. Overall, NLC was non-inferior to RLC with respect to the effectiveness of care when considering all included patients and the csDMARDs subgroups only (Fig. 1 ). In the analysis where missing DAS-28 data were imputed based on the rheumatology team's opinion about the patient's disease status, similar results were observed (Table 4).

Records of patients followed in the NLC model more commonly reported on comorbidities screening and documented patient education compared to the records of patients in the RLC group (Table 4). The groups were most alike in the chart documentation on smoking cessation counseling for current smokers or tobacco users with $84 \%$ of NLC and $80 \%$ of RLC eligible charts having corresponding documentation. However, differences in other process measures were observed between the groups. For example, over $90 \%$ of the NLC charts included documentation on the provided patient education about RA flares and when to contact the clinic in case of a flare, communication to the PCP of the increased cardiovascular risk associated with RA, and patient education about benefits of vaccinations at least once over the follow-up time. In the RLC groups, up to $8 \%$ of charts included documentation on these outcomes.

\section{DISCUSSION}

This non-inferiority study evaluated the quality of care for stable patients with RA in a collaborative NLC model compared to the traditional RLC model using a structured evaluation framework. Overall, NLC was at least as effective as RLC with better documentation of comorbidity screening and provision of patient education.

Our results support findings of previous evaluations and extend them in the context of real-life routine practice conditions, thus, focusing on the 'real-world' effectiveness of NLC models. Consistent with our findings, previous evaluations of NLC models for stable patients with RA [27-29] showed that NLC was non-inferior to RLC with mean differences in DAS-28 (95\% CI) between the groups of $-0.21(-0.53,0.11)$ and $0.04(-0.19,0.27)$ at 12 months [27, 28]. These results were obtained in randomized control trials showing the efficacy of NLC models under ideal conditions rather than their effectiveness in routine clinical practice. 
Furthermore, our evaluation included an assessment of process measures to capture effects associated with complementary aspects of the evaluated NLC model. Few published upto-date qualitative and mixed-methods studies reported that nurses more frequently provided psychosocial support, education about the disease, and screening of comorbidities compared to RLC as perceived by patients and/or members of the rheumatology team $[29,30]$. Consistent with these perceptions, higher rates of documentation of comorbidities screening, patients' education, and wellness assessment were observed in the NLC group across all process measures assessed in our study. Given the reported gaps in addressing the complex needs of patients with RA [21], NLC may present a potential solution to improving the quality of patients' care.

Findings of this study should be considered in the context of the strengths and limitations of the data. The documentation of clinical encounters varied between the groups. In the NLC group, a standardized EMR was used. In the RLC, no standardized data entry was used. Standardized EMRs have the potential to improve the quality of care and its documentation when used in a "meaningful" way [31]. Overall, records of patients in the NLC tended to be more complete in terms of clinical characteristics of patients and processes of care. Comorbidities screening and patient education may have taken place in the RLC group but was less consistently documented. Moreover, due to missing data, the final sample available for the analysis of some outcomes was less than required based on sample size calculations. Nonetheless, results of the additional analysis where the rheumatology team's opinion about the patient's disease status informed imputations of missing disease activity data, were consistent with the complete case analyses.

Amongst other limitations, the retrospective and non-randomized nature of this study should be discussed. Overall, given the retrospective nature of the study, outcomes of this evaluation were limited by the data available. The evaluation was limited to outcome and process measures within dimensions of the effectiveness and appropriateness of care and did not assess patients' experience with care. Nonetheless, an overall high patients' satisfaction with the evaluated nurse-led clinic was previously reported [32].

To identify the comparator group from site B we applied the criteria used to identify eligible patients for NLC at site A. Yet, more patients in the RLC group were treated with biologics agents compared to the NLC group. In the evaluated NLC model, eligible patients were selected based on their disease activity status, and biologic treatment was not an exclusion criterion. However, before the establishment of the evaluated NLC, at site A, some stable patients with inflammatory arthritis treated with biologic agents were already cared for in a nurse-led biologic clinic. This could potentially lead to the observed difference in treatment patterns between the groups. Nonetheless, both groups were selected based on their disease activity status and, as expected, baseline DAS-28 scores were comparable between the NLC and RLC groups $(p=0.21)$. While our study did not aim to compare NLC in patients treated with csDMARDs versus patients treated with biologic agents, results of the sensitivity analysis with csDMARDs subgroups were consistent with the main analysis. These findings suggest that stable disease status rather than the treatment strategy could be a defining factor for identifying patients eligible for the NLC follow-up.

Furthermore, the difference in baseline functional status measure, HAQ-DI, between the groups was statistically significant. Higher HAQ-DI scores in the RLC group could be driven by the inclusion of a larger proportion of patients treated with biologic agents, which could be used more frequently in more severe cases of RA. In the sensitivity analysis, baseline HAQ-DI scores were not statistically significantly different between the csDMARDs subgroups. This could potentially reflect the complexity of clinical decision-making when identifying patients eligible for the nurse-led follow-up, which may be based on the whole picture of the patient's status known to their treating physician rather than the predefined selection criteria. Nonetheless, median baseline HAQ-DI scores in both groups were in the normal to mild functional loss range [33]. 
Additionally, in NLC, it is common practice for nurses to discuss HAQ-DI scores with patients to better differentiate between functional impairment caused by RA versus other comorbidities. If during the conversation, it was determined that the HAQ-DI score was not reflective of RArelated functional impairments, the questionnaire was revised with patient input. Therefore, lower HAQ-DI scores in the NLC group could also be driven by the education provided by nurses.

Lastly, at site A, only consented patients joined the NLC; and only patients who continued their follow-up in the NLC were included in the NLC group. This could potentially introduce a bias due to confounding as patient individual characteristics may influence their preferences for the type of follow-up care [34]. However, the direction of this association is yet to be explored. The available data did not allow us to estimate the number of potentially eligible patients that could be referred to the NLC or the characteristics of patients, which could influence follow-up care preferences. In the future, prospective studies should explore both clinical and personal characteristics of patients who are most likely to benefit from NLC.

Published literature on NLC models often lacks detailed descriptions of the evaluated models, limiting the potential for a broader adoption [13, 15]. Given the importance of the structure of the intervention and contextual factors in the evaluation and implementation of complex interventions as models of care [15], a detailed description of the evaluated model of care represents an important strength of this study. We used the Framework for Reporting Health Service Delivery Models for Managing Rheumatoid Arthritis [35] to discuss details of the NLC and RLC models' implementation, their goals, target population, care providers and their roles, and services provided. Importantly, in contrast with most evaluated NLC models for patients with RA [13], this study evaluated a model which employs registered nurses without an advanced practice designation. Advanced practice nurses (e.g., nurse practitioners, clinical nurse specialists) require additional training and generally are paid a higher salary than registered nurses, which should also be considered when estimating costs of NLC models. Thus, in the light of financial constraints in healthcare systems, a model utilizing registered nurses with additional rheumatology training to their full scope of practice might be particularly attractive to decision-makers. Our non-inferiority study with 1-year follow-up provides a foundation for future evaluations of the model's long-term effectiveness and cost-effectiveness.

\section{CONCLUSIONS}

This study provided real-world evidence that the evaluated NLC model providing protocolized follow-up care for stable patients with RA represents an effective strategy to address the needs of stable patients with RA for ongoing disease monitoring, chronic disease management, education, and support. In the model, registered nurses act in an alternative role to monitor patients' disease activity in lieu of a rheumatologist; and in complementary roles to provide patients comorbidity screening and education, as well as to support standardized clinic documentation and communication with PCPs. In the context of the limited capacity of rheumatologists, NLC is one approach to consider for providing better access to effective and appropriate care for stable patients with RA.

\section{ACKNOWLEDGEMENTS}

The authors acknowledge the contribution of rheumatologists, nurses, staff, and patients at sites A and B for their support with this work.

Funding. During her Ph.D., Dr. Lopatina was funded through the Mitacs Accelerate Internship and Canadian Institutes of Health Research Frederick Banting and Charles Best Canada Graduate Scholarship Doctoral Award. The data collection for this study was funded through Dr. Lopatina's Mitacs Accelerate Internship and Dr. Marshall's Arthur J.E. Child Chair, Rheumatology Outcomes Research in the McCaig Institute of Bone and Joint Health. The 
Rapid Service Fee was funded through Dr. Lopatina's Canadian Institutes of Health Research Frederick Banting and Charles Best Canada Graduate Scholarship Doctoral Award and Dr. Marshall's Arthur J.E. Child Chair, Rheumatology Outcomes Research in the McCaig Institute of Bone and Joint Health.

Authorship. All named authors meet the International Committee of Medical Journal Editors (ICMJE) criteria for authorship for this article, take responsibility for the integrity of the work as a whole, and have given their approval for this version to be published.

Authorship Contributions. All authors contributed to the study conception and design. Data collection was performed by Elena Lopatina, Carolina De la Rossa Jaimes, and Anne Marie Lauf. Data analysis was performed by Elena Lopatina. The first draft of the manuscript was written by Elena Lopatina and all authors commented on previous versions of the manuscript. All authors read and approved the final manuscript.

Disclosures. Elena Lopatina, Deborah A. Marshall, Sharon A. Le Clercq, Tom W. Noseworthy, Esther Suter, Carolina De la Rossa Jaimes, Anne Marie Lauf, Dianne P. Mosher, Claire E. H. Barber have nothing to disclose.

Compliance with Ethics Guidelines. This study was performed in accordance with the Helsinki Declaration of 1964 and its later amendments. The project was approved by the University of Calgary Health Research Ethics Board (REB17-1678). The approval included a waiver of patient consent for this research and publication due to the limited feasibility of obtaining patients' consents for the collection and analysis of retrospective data and the publication of results as well as the minimal risks or discomforts associated with the research.

Data Availability. The datasets generated during and/or analyzed during the current study may be available from the corresponding author on reasonable request.
Open Access. This article is licensed under a Creative Commons Attribution-NonCommercial 4.0 International License, which permits any non-commercial use, sharing, adaptation, distribution and reproduction in any medium or format, as long as you give appropriate credit to the original author(s) and the source, provide a link to the Creative Commons licence, and indicate if changes were made. The images or other third party material in this article are included in the article's Creative Commons licence, unless indicated otherwise in a credit line to the material. If material is not included in the article's Creative Commons licence and your intended use is not permitted by statutory regulation or exceeds the permitted use, you will need to obtain permission directly from the copyright holder. To view a copy of this licence, visit http:// creativecommons.org/licenses/by-nc/4.0/.

\section{REFERENCES}

1. Abhishek A, Doherty M, Kuo C-F, Mallen CD, Zhang W, Grainge MJ. Rheumatoid arthritis is getting less frequent-results of a nationwide population-based cohort study. Rheumatology. 2017;56: 736-44.

2. Marshall DA, Pham T, Faris P, Chen G, O'Donnell S, Barber $\mathrm{CE}$, et al. Determination of rheumatoid arthritis incidence and prevalence in Alberta using administrative health data. ACR Open Rheumatol. 2020;2:424-9.

3. van Onna M, Boonen A. The challenging interplay between rheumatoid arthritis, ageing and comorbidities. BMC Musculoskelet Disord. 2016;17:184.

4. Nikiphorou E, Norton S, Carpenter L, Dixey J, Andrew Walsh D, Kiely P, et al. Secular changes in clinical features at presentation of rheumatoid arthritis: increase in comorbidity but improved inflammatory states. Arthritis Care Res. 2017;69: $21-7$.

5. Smolen JS, Landewé R, Bijlsma JW, Burmester GR, Dougados M, Kerschbaumer A, et al. EULAR recommendations for the management of rheumatoid arthritis with synthetic and biological diseasemodifying antirheumatic drugs: 2019 update. Ann Rheum Dis. 2020;69:964-75. 
6. Bykerk VP, Akhavan P, Hazlewood GS, Schieir O, Dooley A, Haraoui B, et al. Canadian Rheumatology Association recommendations for pharmacological management of rheumatoid arthritis with traditional and biologic disease-modifying antirheumatic drugs. J Rheumatol. 2012;39:1559-82.

7. Singh JA, Saag KG, Bridges SL Jr, Akl EA, Bannuru RR, Sullivan MC, et al. 2015 American College of Rheumatology guideline for the treatment of rheumatoid arthritis. Arthritis Rheum. 2016;68: 1-26.

8. Lacaille D, Anis AH, Guh DP, Esdaile JM. Gaps in care for rheumatoid arthritis: a population study. Arthritis Care Res. 2005;53:241-8.

9. Widdifield J, Bernatsky S, Paterson JM, Thorne JC, Cividino A, Pope J, et al. Quality care in seniors with new-onset rheumatoid arthritis: a Canadian perspective. Arthritis Care Res. 2011;63:53-7.

10. Qian J, Feldman DE, Bissonauth A, Ménard H-A, Panopalis P, Stein M, et al. A retrospective review of rheumatology referral wait times within a health centre in Quebec, Canada. Rheumatol Int. 2010;30: 705-7.

11. Barber CEH, Jewett L, Badley EM, Lacaille D, Cividino A, Ahluwalia V, et al. Stand up and be counted: measuring and mapping the rheumatology workforce in Canada. J Rheumatol. 2017;44: 248-57.

12. Bech B, Primdahl J, Van Tubergen A, Voshaar M, Zangi HA, Barbosa L, et al. 2018 update of the EULAR recommendations for the role of the nurse in the management of chronic inflammatory arthritis. Ann Rheum Dis. 2020;79:61-8.

13. Garner S, Lopatina E, Rankin JA, Marshall DA. Nurse-led care for patients with rheumatoid arthritis: a systematic review of the effect on quality of care. J Rheumatol. 2017;44:757-65.

14. Lempp H, Baggott R, Scott DL, Parker L, Bosworth A, Georgopoulou S, et al. The value, impact and role of nurses in rheumatology outpatient care: critical review of the literature. Musculoskelet Care. 2020;18:245-55.

15. Lopatina E, Donald F, DiCenso A, Martin-Misener R, Kilpatrick K, Bryant-Lukosius D, et al. Economic evaluation of nurse practitioner and clinical nurse specialist roles: a methodological review. Int J Nurs Stud. 2017;72:71-82.

16. De Thurah A, Esbensen BA, Roelsgaard IK, Frandsen TF, Primdahl J. Efficacy of embedded nurse-led versus conventional physician-led follow-up in rheumatoid arthritis: a systematic review and metaanalysis. RMD Open. 2017;3:1-9.
17. Sweeney AMT, McCabe C, Flurey CA, Robson JC, Berry A, Richards P, et al. The patient perspective of nurse-led care in early rheumatoid arthritis: a systematic review of qualitative studies with thematic analysis. J Clin Nurs. 2021;30:145-60.

18. Minnock P, McKee G, Kelly A, Carter SC, Menzies V, $\mathrm{O}^{\prime}$ Sullivan D, et al. Nursing sensitive outcomes in patients with rheumatoid arthritis: a systematic literature review. Int J Nurs Stud. 2018;77:115-29.

19. Harris PA, Taylor R, Minor BL, Elliott V, Fernandez $\mathrm{M}$, O'Neal L, et al. The REDCap consortium: building an international community of software platform partners. J Biomed Inform. 2019;95: 103208.

20. Harris PA, Taylor R, Thielke R, Payne J, Gonzalez N, Conde JG. A metadata-driven methodology and workflow process for providing translational research informatics support. J Biomed Inform. 2009;42:377-81.

21. Barber CEH, Esdaile JM, Martin LO, Faris P, Barnabe $\mathrm{C}$, Guo S, et al. Gaps in addressing cardiovascular risk in rheumatoid arthritis: assessing performance using cardiovascular quality indicators. J Rheumatol. 2016;43:1965-73.

22. Barber CE, Then KL, Bohm V, Hall M, Marshall DA, Rankin JA, et al. Development of a patient-centered quality measurement framework for measuring, monitoring, and optimizing rheumatoid arthritis care in Canada. J Rheumatol. 2021;48:326-34.

23. Kania-Richmond A, Werle J, Robert J. Bone and joint health strategic clinical network: keeping Albertans moving. CMAJ. 2019;191:S10-2.

24. Piaggio G, Elbourne DR, Pocock SJ, Evans SJ, Altman DG, CONSORT Group FT. Reporting of noninferiority and equivalence randomized trials: extension of the CONSORT 2010 statement. JAMA. 2012;308:2594-604.

25. Fransen J, Van Riel PL. The Disease Activity Score and the EULAR response criteria. Rheum Dis Clin North Am. 2009;35:745-57.

26. Ward MM, Guthrie LC, Alba MI. Clinically important changes in individual and composite measures of rheumatoid arthritis activity: thresholds applicable in clinical trials. Ann Rheum Dis. 2015;74: 1691-6.

27. Primdahl J, Wagner L, Holst R, Hørslev-Petersen K, Group AS. The impact on self-efficacy of different types of follow-up care and disease status in patients with rheumatoid arthritis-a randomized trial. Patient Educ Couns. 2012;88:121-8. 
28. Larsson I, Fridlund B, Arvidsson B, Teleman A, Bergman S. Randomized controlled trial of a nurseled rheumatology clinic for monitoring biological therapy. J Adv Nur. 2014;70:164-75.

29. Ndosi M, Lewis M, Hale C, Quinn H, Ryan S, Emery $P$, et al. The outcome and cost-effectiveness of nurse-led care in people with rheumatoid arthritis: a multicentre randomised controlled trial. Ann Rheum Dis. 2014;73:1975-82.

30. Muñoz-Fernández S, Aguilar MD, Almodóvar R, Cano-García L, Fortea S, Alcañiz-Escandell CP, et al. SCORE study: quality indicators for rheumatology nursing clinics. Rheumatol Int. 2017;37:353-61.

31. Menachemi N, Collum TH. Benefits and drawbacks of electronic health record systems. Risk Manag Healthc Policy. 2011;4:47.

32. Thomson S, Howse R, Brown L, Le Clercq S, FifiMah A. Patient satisfaction with a nurse-led stable arthritis clinic in Canada. J Rheumatol. 2019;46:797-797.

33. Maska L, Anderson J, Michaud K. Measures of functional status and quality of life in rheumatoid arthritis: Health Assessment Questionnaire Disability Index (HAQ), Modified Health Assessment Questionnaire (MHAQ), Multidimensional Health Assessment Questionnaire (MDHAQ), Health Assessment Questionnaire II (HAQ-II), Improved Health Assessment Questionnaire (Improved HAQ), and Rheumatoid Arthritis Quality of Life (RAQoL). Arthritis Care Res. 2011;63:S4-13.

34. Laurant MG, Hermens RP, Braspenning JC, Akkermans RP, Sibbald B, Grol RP. An overview of patients' preference for, and satisfaction with, care provided by general practitioners and nurse practitioners. J Clin Nurs. 2008;17:2690-8.

35. O’Donnell S, Li LC, King J, Lauzon C, Finn H, Vlieland TPV. Development of a framework for reporting health service models for managing rheumatoid arthritis. J Clin Rheumatol. 2010;29: 151.

36. Li LC, Badley EM, MacKay C, Mosher D, Jamal SW, Jones A, et al. An evidence-informed, integrated framework for rheumatoid arthritis care. Arthritis Care Res. 2008;59:1171-83.

37. American College of Rheumatology. Advanced Rheumatology Course. [Internet]. http://www. rheumatology.org/Learning-Center/EducationalActivities/View/ID/910. Accessed 12 Feb 2021.

38. D'Agostino RB, Vasan RS, Pencina MJ, Wolf PA, Cobain M, Massaro JM, et al. General cardiovascular risk profile for use in primary care. Circulation. 2008;117:743-53.

39. Adami G, Saag KG. Osteoporosis pathophysiology, epidemiology, and screening in rheumatoid arthritis. Curr Rheumatol Rep. 2019;21:34. 